\title{
Relating eddy correlation sensible heat flux to horizontal sensor separation in the unstable atmospheric surface layer
}

\author{
Xuhui Lee and T. Andrew Black \\ Department of Soil Science, University of British Columbia, Vancouver, Canada
}

\begin{abstract}
Measurements of a scalar flux from an extended surface are frequently made with the eddy correlation technique consisting of a vertical velocity sensor and a sensor for the scalar of interest. In many cases the two sensors have to be mounted with a significant horizontal separation to avoid flow interference. Consequently, the technique will underestimate the scalar flux. This paper addresses the issue of flux underestimation due to this separation. A model is developed in the framework of Monin-Obukhov similarity for the spatial covariance of vertical velocity and air temperature in the unstable surface layer. It allows the underestimation of sensible heat flux to be assessed using information on separation orientation relative to wind direction, atmospheric stability, measurement height, and separation distance. The coefficient in the model is evaluated with observations made over a potato field and a clover field. The principles established here should also be applicable to fluxes of scalars other than sensible heat.
\end{abstract}

\section{Introduction}

Measurements of the vertical fluxes of atmospheric scalar constituents are important in studies of processes controlling the exchange of these scalars between land surfaces and the atmosphere. Over an extensive and uniform surface on flat terrain these measurements are frequently made with the eddy correlation technique consisting of a fast response velocity sensor for measuring the vertical velocity component and a sensor for the scalar of interest. In many cases the two sensors have to be mounted with a significant horizontal separation to avoid flow interference because either the scalar sensor is rather bulky or it has to be confined in a weather-proof enclosure to allow long-term operation. As a result of the separation this technique underestimates the flux. Previously, a few workers have expressed concern about this issue (see below). Their work appears to indicate that the degree of flux underestimation depends on separation distance, measurement height, atmospheric stability and separation orientation relative to wind direction. However, as pointed out in the next section of this paper, there is no theory that relates the underestimation to all these factors to allow quantitative assessment.

The main purpose of this paper is to develop a model for assessing the underestimation of eddy correlation sensible heat flux due to horizontal sensor separation in the unstable atmospheric surface layer. It is also hoped that the present study will improve our understanding of the horizontal turbulent structure near the ground surface. We chose to study sensible heat for the reason that measurements of temperature fluctuations can be easily made at multiple points with thermocouples. However, the principles established here should also be applicable to scalars other than sensible heat, based on the similarity of scalar transport processes in the atmosphere [Hill, 1989].

Copyright 1994 by the American Geophysical Union.

Paper number 94JD00942.

0148-0227/94/94JD-00942\$05.00
Prior to the analysis it is useful to review previous studies relevant to this work, which is done in section 2 . Section 3 then establishes a model in the framework of MoninObukhov similarity theory for assessing the flux underestimation due to spatial separation. Experimental methods are described in section 4 . In section 5 the constants that appear in the model are evaluated from the observations. Finally, in section 6 the implications of the model are briefly discussed.

\section{Review}

The eddy correlation technique measures the flux of sensible heat by forming a covariance between vertical velocity $(w)$ and temperature $(T), \overline{w^{\prime}(\vec{x}) T^{\prime}(\vec{x}+\vec{r})}$, where $w^{\prime}(\vec{x})$ and $T^{\prime}(\vec{x}+\vec{r})$ are fluctuations of vertical velocity at a horizontal position $\vec{x}$ and temperature at $\vec{x}+\vec{r}$ and the overbar denotes temporal (Reynolds) averaging. Since horizontally homogeneous surfaces are the concern of this study, $\overline{w^{\prime}(\vec{x}) T^{\prime}(\vec{x}+\vec{r})}$ is independent of $\vec{x}$ and can be denoted as $H(\vec{r})$. Ideally $w^{\prime}$ and $T^{\prime}$ should be measured at the same point $(\vec{r}=0)$, and the covariance $(H(0))$ is the kinematic sensible heat flux density. Otherwise $H(\vec{r})$ will be different from $H(0)$.

Because $H(\vec{r})$ is a spatial covariance, in principle its conservation equation can be constructed from the NavierStokes and heat conservation equations, in a manner similar to that of deriving the conservation equations of the spatial covariances of velocity components or temperature [Hinze, 1975; Tatarskii, 1971]. Finding solutions to these conservation equations is difficult because of the closure problem (there are more unknown variables than the governing equations) and it is only possible in highly simplified situations. For example, provided that turbulence is isotropic, analytical expressions have been derived for the spatial covariances of velocity components and those of temperature [Hinze, 1975]. The assumption of isotropy, however, leads to zero covariance between velocity and any of the scalars [Tatarskii, 1971] and is not appropriate at all for $w-T$ covariance. 
Instead of assuming that the flow is completely isotropic, Moore [1986] used the property of local isotropy in the inertial subrange in the wavelength domain. He defined a spectral transfer function, $T_{s}$, associated with sensor separation, in a similar manner as done to assess the effect of line averaging [Silverman, 1968; Kristensen and Fitzjarrald, 1984]. He argued that if separation distance $r(=|\vec{r}|, m)$ is small and only affects turbulence fluctuations in the inertial subrange, the velocity cross spectra obtained for isotropic turbulence can be used to define $T_{s}$, which has the following working description for lateral separation:

$$
T_{s}(f)=\exp \left(-9.9 f^{1.5}\right),
$$

where $f=n r / u, n$ being natural frequency $(\mathrm{Hz})$ and $u$ wind speed $(\mathrm{m} / \mathrm{s})$. Hence

$$
H(r)=H(0) \int_{0}^{\infty} T_{s}(f) S_{w T}(n) \mathrm{d} n
$$

where $S_{w T}$ is the cospectrum of sensible heat flux in the surface layer.

For longitudinal separation, Taylor's hypothesis of frozen turbulence will result in unrealistic longitudinal coherence in isotropic turbulence [Kristensen and Jensen, 1979]. Consequently, the concept of eddy turnover time has to be incorporated into spectral characteristics [Kristensen, 1979]. Assuming that $r / u$ is small compared to eddy turnover time and the quadrature spectrum is negligible, Moore [1986] arrived at the following transfer function for longitudinal separation:

$$
T_{s}(f)=\cos (2 \pi f) \text {. }
$$

(On the other hand, G. W. Thurtell [see Heikinheimo, 1986] believed that (3) should represent the transfer function for lateral separation.)

It can be shown from (1) (or (3)), (2), and the expression for $S_{w T}$ in the neutral and unstable surface layer [Kaimal et al., 1972; Kristensen and Fitzjarrald, 1984] that the ratio, $H(\vec{r}) / H(0)$, is a function of $r / z_{m}$, where $z_{m}=z-d$, with $z$ the height above the ground surface and $d$ the displacement height, and is independent of wind speed. The $r / z_{m}$ dependence has somewhat been confirmed by a few experiments [Heikinheimo, 1986; Koprov and Sokolov, 1973]. However, there are three significant problems with the above transfer function approach: it implies similarity between velocity components and $w-T$ spatial cospectra, which, for the reason that will become clear later, is not valid; it is not appropriate when separation $r$ is large; and it does not relate $w-T$ covariance to separation orientation or stability.

Monin-Obukhov similarity, on the other hand, may provide a more promising tool than the above approaches for predicting turbulence structure over horizontally homogeneous surfaces. For example, it has been used for studying the behavior of the structure function, $D_{q q}$, of a certain quantity, $q$ [Tatarskii, 1971], which is defined as

$$
D_{q q}=\overline{[q(\vec{x})-q(\vec{x}+\vec{r})]^{2}} .
$$

At separation distances of inertial subrange scale, the temperature or humidity structure function has the form

$$
D_{q q}=C_{q}^{2} r^{2 / 3},
$$

where the proportionality factor, $C_{q}^{2}$, called the structure parameter, is independent of separation distance, $r$. The velocity structure function also follows the $2 / 3$ power law expression, but the direction of separation is important [Tatarskii, 1971]. It is well established now that $C_{q}^{2}$ can be related to surface layer parameters in the framework of Monin-Obukhov similarity. A good illustration of this is the expression for the temperature structure parameter derived from dimensional analysis

$$
C_{T}^{2}=T_{*}^{2} z_{m}^{-2 / 3} g\left(z_{m} / L\right),
$$

where $T_{*}$ is a temperature scale defined as the ratio of the kinematic sensible heat flux to the friction velocity $\left(u_{*}\right)$

$$
T_{*}=-H(0) / u_{*},
$$

and $L$ is the Monin-Obukhov length scale [Wyngaard et al., 1971]. With the assumption of horizontal homogeneity a little manipulation of (4)-(7) yields an expression for the spatial temperature covariance at small $r$

$\overline{T^{\prime}(x) T^{\prime}(x+r)}=\overline{T^{\prime 2}(x)}\left[1-\frac{T_{*}^{2}}{2 \overline{T^{\prime 2}}}\left(\frac{r}{z_{m}}\right)^{2 / 3} g\left(\frac{z_{m}}{L}\right)\right]$,

where $x=|\vec{x}|$. The function $g$ was evaluated experimentally by Wyngaard et al. [1971] and was derived by Panofsky and Dutton [1984] from balance equations of turbulence kinetic energy and temperature fluctuations. Since $T_{*}^{2} / \overline{T^{\prime 2}}$ is also a function of $z_{m} / L$ only, (8) reveals that temperature covariance can be calculated from the measurements of $L$. It is tempting to assume that spatial decays of $T$ and $w-T$ covariances are identical so that

$$
H(r)=H(0)\left[1-\frac{T_{*}^{2}}{2 T^{\prime 2}}\left(\frac{r}{z_{m}}\right)^{2 / 3} g\left(\frac{z_{m}}{L}\right)\right] .
$$

This, however, will result in errors. In an unstable surface layer, the decay of $w$ spatial covariance with $r$ is expected to be faster than that of $T$ covariance, as the energy-containing range occurs at larger wavelengths for the $T$ power spectrum than for the $w$ spectrum. This difference in the two decay rates was observed by Koprov and Sokolov [1973], who also showed that the decay of $w-T$ covariance was in between the two. But it is possible, as discussed in the next section, to develop a model relating $w-T$ covariance to $L$ in a manner similar to that done for $T$ covariance.

In the past, several studies have addressed experimentally the issue of $w-T$ spatial covariance. Leuning et al. [1982] observed that increasing the lateral separation distance between the temperature and vertical velocity sensors of their eddy correlation system from 0.05 to $0.45 \mathrm{~m}$ reduced the measured sensible heat flux by about $6 \%$ at a height of 2 $\mathrm{m}$ in the daytime. Based on limited observations, Koprov and Sokolov [1973] found that the dependence of $w-T$ covariance on lateral separation in slightly unstable conditions $(z / L=-0.02)$ could be described with the following equation:

$$
H(r) / H(0)=\exp \left(-r / z_{m}\right) .
$$

This equation was used by Heikinheimo [1986] as one of the means for estimating the loss of eddy correlation fluxes due to lateral sensor separation. The prediction of (10) appeared to agree with his observations, although the stability during 
his experiment was probably not neutral (see section 6). These experimental studies dealt with specific cases. The question regarding the influence of wind direction and stability still remains unanswered.

In next section, a model will be developed in the context of Monin-Obukhov similarity to allow the assessment of the spatial decay of $w-T$ covariance from the measurements of all controlling factors.

\section{Model}

\subsection{Constraint at Small Separation Distance}

Tatarskii [1971] derived a one-dimensional spectral density function from the structure function (4). By equating this spectral density function to the inertial subrange spectra, Wyngaard and colleagues [Wyngaard et al., 1971; Wyngaard and Clifford, 1978; Wyngaard and LeMone, 1980] obtained expressions for structure parameters of temperature, humidity, and velocity in the atmospheric surface layer. Alternatively, one can use similarity arguments to arrive at the same results without knowing the details of the spectral dynamics [Tatarskii, 1971; Hill, 1989].

By analogy with (4), let us define a cross-structure function for $w-T$ as

$$
D_{w T}=\overline{[w(\vec{x})-w(\vec{x}+\vec{r})][T(\vec{x})-T(\vec{x}+\vec{r})]} .
$$

Apparently, the direction of separation is important for $D_{w T}$. Because turbulence is considered to be horizontally homogeneous, (11) can be written as

$$
H(\vec{r})=H(0)-D_{w T} / 2,
$$

where $\boldsymbol{H}(\vec{r})=H(-\vec{r})$ is assumed. Wyngaard and Cote [1972] proposed a model for the $w-T$ cospectrum in the inertial subrange that is in excellent agreement with observations. They postulated that the cospectrum depends on vertical potential temperature gradient $(\partial \theta / \partial z)$, dissipation rate of turbulent kinetic energy $(\varepsilon)$, and wavelength. It can be deduced from this model that for $r$ in the inertial subrange, $D_{w T}$ should depend on $\partial \theta / \partial z, \varepsilon$, and $r$. Dimensional analysis requires that

$$
D_{w T}=2 k^{4 / 3} \alpha(\delta)|\partial \theta / \partial z| \varepsilon^{1 / 3} r^{4 / 3} .
$$

Here $k(=0.4)$ is the von Karman constant and $\alpha(\delta)$ is a wind direction dependent coefficient, with $\delta$ defined such that it varies between $0^{\circ}$ when wind is parallel to the direction of separation (longitudinal separation) and $90^{\circ}$ when wind is perpendicular to the direction of separation (lateral separation). Thus $D_{w T}$ does not follow the $2 / 3$ power law of (5) or the $1 / 1$ power law as suggested by (10) at small $r$.

It is known that [Panofsky and Dutton, 1984]

$$
\begin{gathered}
\frac{\partial \theta}{\partial z}=\frac{T_{*}}{k z_{m}} \phi_{h}\left(\frac{z_{m}}{L}\right), \\
\varepsilon=\frac{u_{*}^{3}}{k z_{m}} \phi_{\varepsilon}\left(\frac{z_{m}}{L}\right),
\end{gathered}
$$

where the similarity functions have the forms $(L<0)$

$$
\phi_{h}=\left(1-16 z_{m} / L\right)^{-1 / 2},
$$

$$
\phi_{\varepsilon}=1-z_{m} / L .
$$

Substituting (14)-(17) into (13) and using (7) and (12) yields

$$
H(\vec{r})=H(0)\left[1-\beta\left(\delta, z_{m} / L\right)\left(r / z_{m}\right)^{4 / 3}\right],
$$

where

$$
\beta\left(\delta, z_{m} / L\right)=\alpha(\delta)\left(1-16 z_{m} / L\right)^{-1 / 2}\left(1-z_{m} / L\right)^{1 / 3} .
$$

This completes the derivation of the constraint of $w-T$ covariance at small $r$. The coefficient $\alpha(\delta)$ will be determined experimentally (section 5.2).

\subsection{Constraint at Large Separation Distance}

The $w-T$ covariance should approach zero as separation distance increases

$$
H(\vec{r}) / H(0) \rightarrow 0 \quad \text { as } r \rightarrow \infty .
$$

The exact form of $H$ at large $r$ is not clear. Here we choose to use the following exponential function:

$$
H(\vec{r})=H(0) \exp \left[-\beta\left(\delta, z_{m} / L\right)\left(r / z_{m}\right)^{4 / 3}\right] .
$$

The advantage of this function lies in its simplicity and its ability to match constraints for both small and large separation distances (equations (18) and (20)). As discussed in section 5, (21) fits rather well with the observed behavior of $w$ - $T$ covariance. With a separation distance in the inertial subrange, (21) differs only slightly from (18) (section 6).

\section{Experimental Methods}

\subsection{Site Descriptions}

The experiments were conducted in a potato field and a clover field located on level delta land at the mouth of the Fraser River in Delta, British Columbia, Canada, in the summer of 1992. The potato crop was planted on E-W running ridges $0.20 \mathrm{~m}$ high, $0.41 \mathrm{~m}$ wide, and spaced $0.84 \mathrm{~m}$ apart. The crop was near maturity. The canopy was very dense and the height of the stand, measured from the bottom of the ridges, was $0.76 \mathrm{~m}$. An open-lattice triangular instrument tower $0.25 \mathrm{~m}$ wide was positioned near the SE corner of the field, which was very extensive. The roughness length, estimated from wind speed measured at two heights near-neutral stability, was about $6 \mathrm{~cm}$.

The clover field was $500 \mathrm{~m}(\mathrm{~N}-\mathrm{S}) \times 200 \mathrm{~m}(\mathrm{E}-\mathrm{W})$. To the east of the clover field was a potato field, to the west a bare field, to the north a field of beans in the early stages of development, and to the south a farmhouse and a paved road. The clover canopy was $10 \mathrm{~cm}$ tall and covered about $10 \%$ of the ground surface. The rest of the surface was covered by dry plant residue from the harvest of the previous pea crop, resulting in a significant mulching effect. The instrument tower was positioned at the center of the field. The estimated roughness length was about $3 \mathrm{~cm}$.

\subsection{Instrumentation}

The same apparatus was used for both field experiments. The key instrumentation included a one-dimensional (vertical) sonic anemometer/thermocouple (Campbell Scientific, Incorporated, model CA27, Logan, Utah; unit 1138) and two linear arrays of thermocouples oriented perpendicular to each other, called TX and TY, each consisting of five sensors at various distances from the sonic path (Figure 1). 


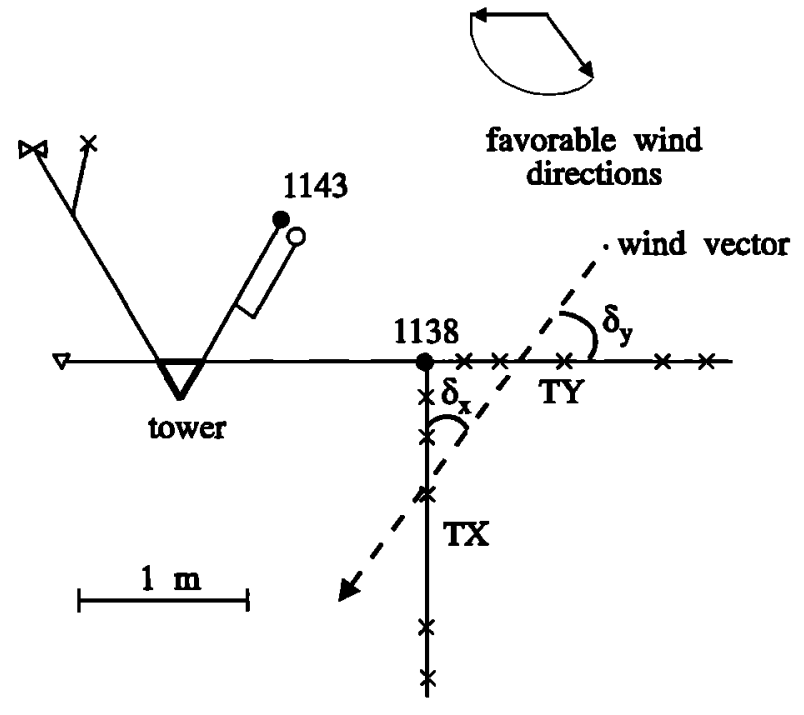

Figure 1. Plan view of the instrument layout for the experiments in the potato and clover fields in Delta, 1992: onedimensional sonic anemometer/thermocouple (solid circle), thermocouple (crosses), cup anemometer (bow tie), fast response hygrometer (open circle), and wind vane (open triangle). Also shown are the angle between horizontal wind vector and each of the two thermocouple arrays (TX and TY). Refer to Table 1 for instrument heights and Table 2 for array separation distances.

All thermocouples were made by cross-welding chromel and constantan wires. The diameter of the wires used for the arrays was $26 \mu \mathrm{m}$ and that for the sonic anemometer was 13 $\mu \mathrm{m}$. The distance between the center of the sonic anemometer path and the thermocouple of unit $1138\left(r_{0}\right)$ was $3.5 \mathrm{~cm}$ and was in the direction of TY. Both arrays were mounted at the height of the center of the sonic path. They were operated at one height $(3.35 \mathrm{~m})$ over the potato field and two heights (1.54 and $3.01 \mathrm{~m}$ ) over the clover field (Table 1). The array thermocouples were supported on two $0.6-\mathrm{cm}$ diameter stainless steel rods. Their measurement junctions were about $15 \mathrm{~cm}$ above the rods so the effect of flow interference on the measurements was minimal.

Signals from unit 1138 and the arrays were sampled at 5 $\mathrm{Hz}$ with a data logger (Campbell Scientific, Incorporated, model 21X with extended software II). The reference junctions for the array thermocouples were at the terminal strip of the data logger. Statistics were calculated on line over

Table 1. Instrument Heights for the Experiments in the Potato and Clover Fields in Delta, 1992

\begin{tabular}{llcccccc}
\hline & & & & \multicolumn{3}{c}{$R i$} & \\
\cline { 3 - 6 } Field & \multicolumn{1}{c}{ DX, TY } & & & & \\
& & 1138 & 1143 & $z_{1}$ & $z_{2}$ & Dir \\
\hline Potato & July 20-26 & 3.35 & 3.50 & 1.57 & 2.91 & 3.35 \\
Clover & July 28 to Aug. 1 & 1.54 & 3.37 & 1.37 & 2.72 & 3.62 \\
& Aug. 1-4 & 3.01 & & & &
\end{tabular}

Heights are given for one-dimensional sonic anemometer/ thermocouple units (1138 and 1143), thermocouple arrays (TX and TY), wind speed and temperature sensors for measuring Richardson number $(R i)$, and wind vane (Dir). For the potato field all heights were measured from the bottom of the ridge. Heights are in meters. 30-min intervals. These were the variances of and covariance between vertical velocity and air temperature from unit 1138 and covariances between each of the two signals from unit 1138 and those of the array thermocouples.

A second eddy correlation unit was mounted at a level above unit 1138 (Table 1). It consisted of a one-dimensional sonic anemometer/thermocouple (1143) of the same type as unit 1138 and a fast response krypton hygrometer (Campbell Scientific, Incorporated, model K20). A second $21 \mathrm{X}$ data logger sampled signals from this unit at $5 \mathrm{~Hz}$ and calculated fluxes of sensible and latent heat over 30 -min intervals. These measurements served as, among other things, a check of the performance of unit 1138. Air temperature and wind speed were measured at two levels with thermocouples of the same type as those of the arrays and sensitive cup anemometers (Thornthwaite Associates, Centerton, New Jersey, model 901-LED), for the purpose of determining the gradient Richardson number. Wind direction was monitored with a wind vane (Met-One, Incorporated, Grants Pass, Oregon, model 024A). Supplementary measurements included net radiation flux, heat flux into the soil, and wet and dry bulb temperature.

After the completion of the two experiments, a threedimensional sonic anemometer/thermometer (Applied Technologies, Incorporated, Boulder, Colorado, model SWS-211/ 3V) was operated at a height of 1.42 over the clover field from August 18-20, 1992, with the two-level thermocouples and cup anemometers still in operation. The MoninObukhov length scale, $L$, was calculated from the fluxes of momentum and sensible heat measured with this unit after the momentum flux was corrected for the shadow effect of the three-dimensional anemometer probe [Lee, 1992].

\subsection{Data Processing}

In the potato field the range of favorable wind directions was $250^{\circ}-300^{\circ}$, with fetches of $400-700 \mathrm{~m}$. In the clover field the range was $120^{\circ}-270^{\circ}$ when the arrays were at the $1.54-\mathrm{m}$ height and $150^{\circ}-210^{\circ}$ when they were at the $3.01-\mathrm{m}$ height. The corresponding fetches were 100-270 and $250-270 \mathrm{~m}$. Tower interference with the measurements was minimal when wind direction was in these ranges (Figure 1). Only runs with favorable wind direction were selected for the following analysis:

The gradient Richardson number $(R i)$ was calculated from

$$
R i \cong \frac{g}{\bar{T}} \frac{\theta_{2}-\theta_{1}}{\left(u_{2}-u_{1}\right)^{2}}\left(z_{2}-z_{1}\right),
$$

where $g$ is the acceleration due to gravity, $\bar{T}$ is the air temperature averaged over heights $z_{1}$ and $z_{2}$ (Table 1), and $\theta_{i}$ and $u_{i}(i=1,2)$ are the potential temperature and wind speed at height $z_{i}$. Ri calculated from (22) is for the geometric mean height above the displacement height $(d)$ for the two measurement levels $\left(z_{1}\right.$ and $\left.z_{2}\right), z_{g}=\left[\left(z_{1}-d\right)\left(z_{2}-d\right)\right]^{0.5}$. (One reviewer brought the work of Arya [1991] to our attention. Arya implied that $R i$ at height $z_{g}$ be calculated as $R i \cong$ $(g / \bar{T})\left(\theta_{2}-\theta_{1}\right) /\left(u_{2}-u_{1}\right)^{2} z_{g} \ln \left[\left(z_{2}-d\right) /\left(z_{1}-d\right)\right]$, which is consistent with Sellers [1965, p. 153]. For our height configuration (Table 1) the difference between this formula and (22) is insignificant.) Since $R i$ is proportional to height in the surface layer under unstable conditions [Businger, 1966; Pandolfo, 1966], the following equation is used to obtain $R i$ for the height of the arrays, $z_{m}$ : 


$$
R i_{z}=\frac{z_{m}}{z_{g}} R i .
$$

$R i_{z}$ is then related to the Monin-Obukhov length scale as [Businger, 1966; Pandolfo, 1966]

$$
\frac{z_{m}}{L}=R i_{z}
$$

Thus the parameter, $z_{m} / L$, which appears in the similarity functions, can be replaced by $R i_{z}$.

Kinematic sensible heat flux was calculated from the gradient measurements (Panofsky and Dutton [1984], combination of their equations $6.8 .4,6.9 .3$, and 6.10.2),

$$
H(0)=-\frac{k^{2}(z-d)^{2}}{\left[\phi_{m}\left(R i_{z}\right)\right]^{3}} \frac{\partial u}{\partial z} \frac{\partial \theta}{\partial z},
$$

or

$H(0)=-\frac{k^{2}}{\left[\phi_{m}(R i)\right]^{3}} \frac{\left(u_{2}-u_{1}\right)\left(\theta_{2}-\theta_{1}\right)}{\left[\ln \left(z_{2}-d\right)-\ln \left(z_{1}-d\right)\right]^{2}}$,

where $\phi_{m}$ is the similarity function for momentum flux and is related to $\phi_{h}$ as

$$
\phi_{m}=\phi_{h}^{1 / 2} \text {. }
$$

Because of the sparseness of the canopy, $d$ was set to zero for the clover field. For the potato field it was estimated to be $0.76 \mathrm{~m}$ (section 5.1). The calculations using (25) were compared with $w-T$ covariance directly measured with eddy correlation unit 1138 to check the consistency among measurements.

Let $\beta_{x}$ and $\beta_{y}$ be the decay coefficients for TX and TY, respectively. It follows from (21) that

$$
\ln \left[H(r) / H\left(r_{0}\right)\right]=-\beta_{y}\left[\left(r / z_{m}\right)^{4 / 3}-\left(r_{0} / z_{m}\right)^{4 / 3}\right],
$$

for array TY, where $H\left(r_{0}\right)$ is the $w-T$ covariance measured with unit 1138 with a slight separation distance, $r_{0}(=3.5$ $\mathrm{cm})$ in the direction of TY, and $H(r)$ was measured with the

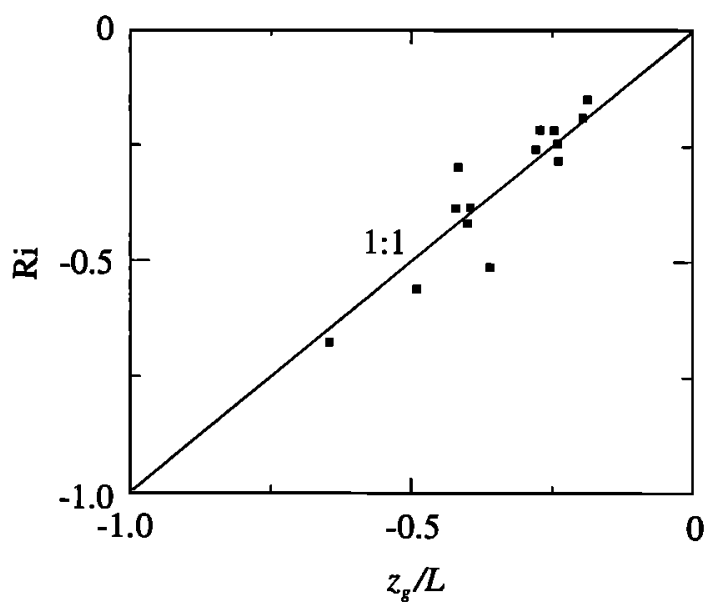

Figure 2. Comparison of the gradient Richardson number $(R i)$ and Monin-Obukhov parameter $\left(z_{g} / L\right)$ over the clover field in Delta, 1992.
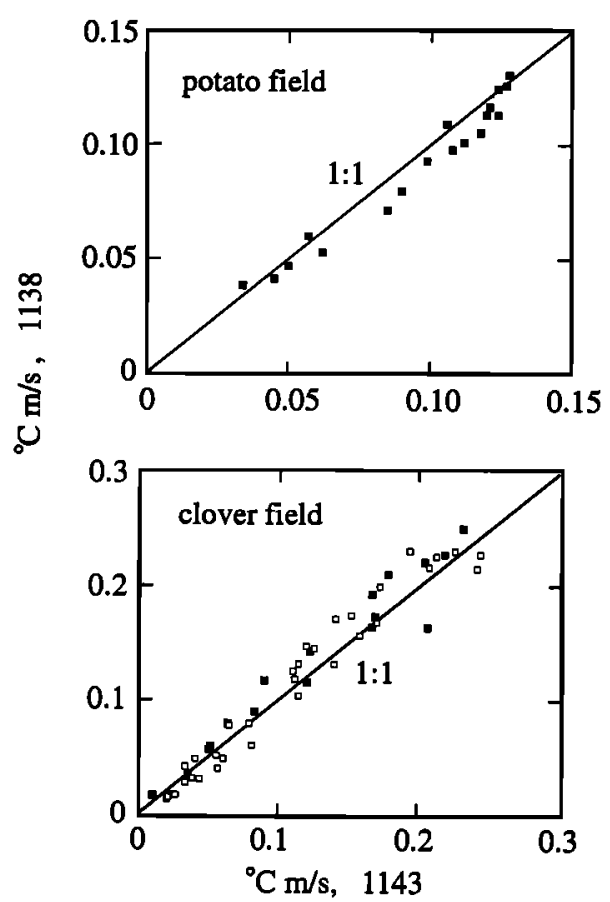

Figure 3. Comparison of $w-T$ covariance $\left({ }^{\circ} \mathrm{C} \mathrm{m} / \mathrm{s}\right)$ measured with the one-dimensional sonic anemometer/thermocouple units 1138 and 1143 over the clover (1138 at height $z=1.54$ (open squares) and $3.01 \mathrm{~m}$ (solid squares) and potato field in Delta, 1992.

sonic anemometer of unit 1138 and the thermocouple of TY at separation $r$ from the anemometer. Similarly for array TX

$$
\begin{aligned}
\ln \left[H(r) / H\left(r_{0}\right)\right] & =-\beta_{x}\left(r / z_{m}\right)^{4 / 3}+\beta_{y}\left(r_{0} / z_{m}\right)^{4 / 3} \\
& \cong-\beta_{x}\left[\left(r / z_{m}\right)^{4 / 3}-\left(r_{0} / z_{m}\right)^{4 / 3}\right] .
\end{aligned}
$$

Therefore $\beta_{x}$ and $\beta_{y}$ were evaluated by linearly regressing $\ln$ $\left[H(r) / H\left(r_{0}\right)\right]$ against $\left(r / z_{m}\right)^{4 / 3}-\left(r_{0} / z_{m}\right)^{4 / 3}$ for individual runs using measurements from each of the two arrays.

\section{Results}

\subsection{Comparison of Measurements}

There was good agreement between $R i$ calculated from (22) and $z_{g} / L$ from the three-dimensional sonic unit (Figure 2). This confirmed (24) and indicated that our technique for determining $R i$ was reliable. Good agreement was also achieved between the values of $w-T$ covariance $\left(H\left(r_{0}\right)\right)$ measured with the two one-dimensional units over both fields. The scatter in the plot was mostly within $10 \%$ of the 1:1 line (Figure 3).

The kinematic sensible heat flux, $H(0)$, calculated from the gradient measurements (equation (25)) was compared with $w-T$ covariance, $H\left(r_{0}\right)$, measured directly with eddy correlation unit 1138 (Figure 4). This comparison provides a rigorous check of our measurement system. No correction was made to $H\left(r_{0}\right)$ to account for the separation distance $r_{0}$. Our calculation based on the model of $w-T$ covariance indicated that this would only cause a very small error, typically $0.5 \%$. As shown in Figure 4 , the scatter for the clover field was rather large but overall was about $1: 1$ line.

The situation for the potato field was rather complex 


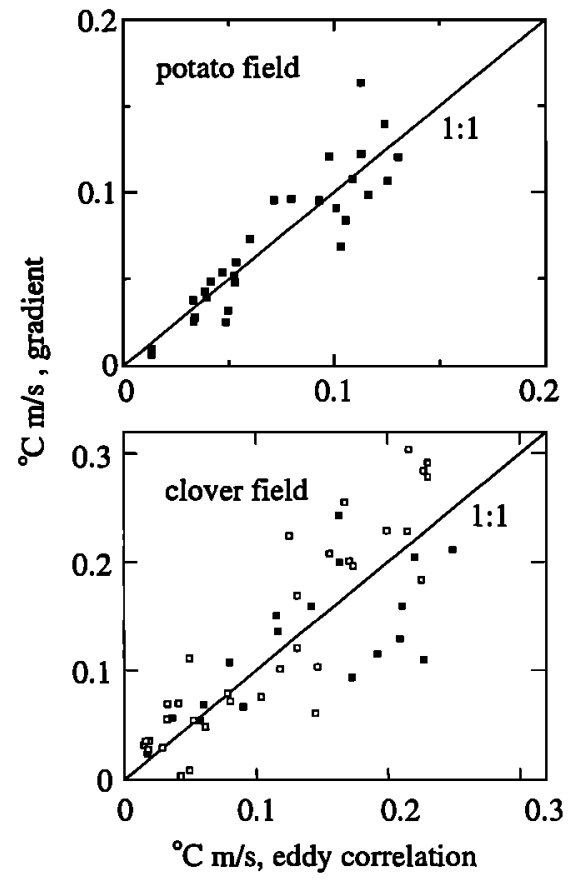

Figure 4. Comparison of the kinematic sensible heat flux $\left({ }^{\circ} \mathrm{C}\right.$ $\mathrm{m} / \mathrm{s}$ ), calculated from gradient measurements, and $w-T$ covariance measured with the eddy correlation technique using unit 1138 over the potato and over the clover field (1138 at $z=1.54$ (open squares) and $3.01 \mathrm{~m}$ (solid squares)) in Delta, 1992. The displacement height $d$ was 0 for the clover field and was determined to be $0.76 \mathrm{~m}$ for the potato field.

because of the unknown parameter, $d$. Using the conventional value of the ratio, $d / h=0.7$ [Jarvis et al., 1976], where $h(=0.76 \mathrm{~m})$ is the height of the stand, we found that $H(0)$ from the gradient measurements was $47 \%$ higher than $H\left(r_{0}\right)$ from the eddy correlation measurements. This was unlikely a result of the roughness sublayer effect because it would have resulted in an underestimation of $\phi_{m}$ compared to that calculated from (16) and (26) [Garratt, 1978], and hence the neglect of the effect would have caused an underestimate of sensible heat flux from (25). To produce good agreement between the two techniques, a value of 0.76 $\mathrm{m}$ for $d$, or $d=h$, was required (Figure 4). This value is rather high, but it is believed to be reasonable because the potato canopy was very dense and completely closed. The dominant part of the sensible heat flux is expected to come from the thin layer very near the top of the stand. The displacement height, if interpreted as the average height of the source of sensible heat [Lee and Black, 1993], should therefore be close to the stand height. This $d$ value was used in the subsequent analysis of the $w-T$ covariance data from the potato field. Sensitivity calculations indicated that the uncertainty in the $d$ value would cause only a very small error in the analysis: for the typical value of $R i=-0.2$, the change of $d / h$ from 0.7 to 1.0 would only increase $H(\vec{r}) / H(0)$ from (21) by less than $3 \%$ for all the separation distances in the potato field experiment.

\subsection{Relating Decay Coefficient $\beta$ to Wind Direction and Stability}

Let $\delta_{x}$ and $\delta_{y}$ be the wind angles for arrays TX and TY, respectively, and note that $\delta_{x}+\delta_{y}=90^{\circ}$ (Figure 1). Figure 5 shows the ratio of the decay coefficient for TX to that for
TY, $\beta_{x} / \beta_{y}$, determined from the observations (section 4.3), plotted against $\sin \delta_{x}$. The influence of the wind angle on the spatial behavior of $w-T$ covariance can be clearly seen: the decay in the lateral direction is about twice that in the longitudinal direction. This is because turbulent eddies tend to be elongated in the mean wind direction in the surface layer [Panofsky and Dutton, 1984]. Since for a specific run, stability is the same for both arrays, it follows from (19) that the ratio is a function of $\delta_{x}$ or $\delta_{y}$ only. To further quantify the effect of the wind angle, we propose that turbulent eddies have an elliptical shape such that

$$
\alpha(\delta)=a\left(\cos ^{2} \delta+b \sin ^{2} \delta\right)^{2 / 3},
$$

where $a$ and $b$ are two constants. Hence

$$
\frac{\beta_{x}}{\beta_{y}}=\frac{\left(\cos ^{2} \delta_{x}+b \sin ^{2} \delta_{x}\right)^{2 / 3}}{\left(b \cos ^{2} \delta_{x}+\sin ^{2} \delta_{x}\right)^{2 / 3}} .
$$

With a value of $b=2.4$, (30) fits well the observations in Figure 5.

A little manipulation of (19) and (29) yields

$$
\begin{array}{r}
\left(\beta_{x}^{3 / 2}+\beta_{y}^{3 / 2}\right)^{2 / 3}=a(1+b)^{2 / 3}\left(1-16 R i_{z}\right)^{-1 / 2} \\
\cdot\left(1-R i_{z}\right)^{1 / 3} .
\end{array}
$$

Figure 6 shows $\left(\beta_{x}^{3 / 2}+\beta_{y}^{3 / 2}\right)^{2 / 3}$ plotted against $R i_{z}$. As indicated by this figure, the decay of $H$ was greatly influenced by stability. There was considerable scatter in nearneutral conditions, which was a result of large relative errors arising from the measurements of $\boldsymbol{H}$ of small magnitudes. The trend is clearly predicted by (31): lower decay occurred in more unstable conditions. Based on the data presented in Figure $6, a(1+b)^{2 / 3}$ was evaluated to be 2.67 or $a=1.18$.

In summary the decay coefficient $(\beta)$ can be expressed as follows:

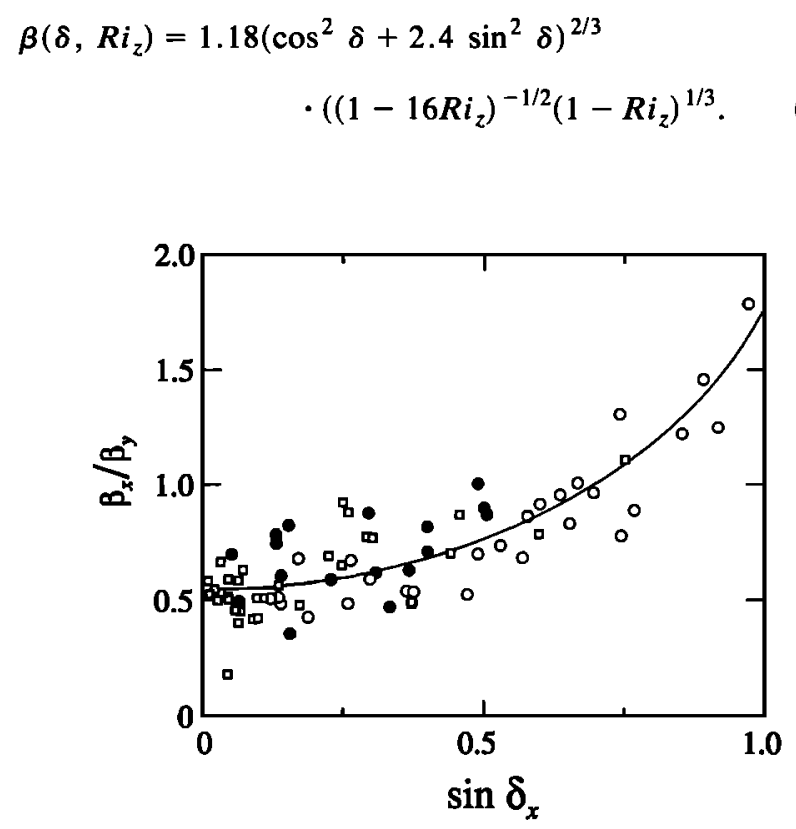

Figure 5. The ratio of decay coefficient of array TX to that of TY $\left(\beta_{x} / \beta_{y}\right)$ plotted against the sine of the angle between array TX and the horizontal wind vector $\left(\delta_{x}\right)$ over the potato (squares, $z-d=2.59 \mathrm{~m}$ ) and clover (open circles, $z-$ $d=1.54 \mathrm{~m}$; solid circles, $z-d=3.01 \mathrm{~m}$ ) fields in Delta, 1992. The solid curve represents equation (30) with $b=2.4$. 


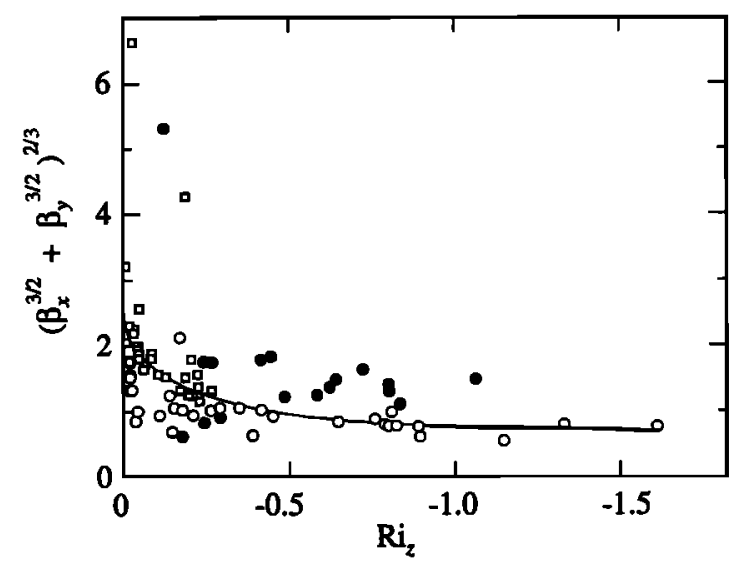

Figure 6. The decay coefficient plotted as a function of Richardson number $\left(R i_{z}\right)$ over the potato and clover fields in Delta, 1992. The solid curve represents equation (31) with $a=$ 1.18. Other variables and symbols are the same as in Figure 5.

\subsection{Comparison of Calculations of (21) and (32) With Observations}

Figure 7 compares the observed decay of $w-T$ covariance with that calculated using (21) and (32) for 2 days, one for each of the fields. These days were selected for comparison because continuous measurements were made throughout the day and there were wide ranges of wind angle $(\delta)$ and stability $\left(R i_{z}\right)$. The influence of stability is obvious. For example, runs 1330 and 1730 PST in the clover field had similar wind angles, but run 1330 experienced a smaller decay of $w-T$ covariance because the air was more unstable. The influence of wind direction is best shown by Figure 7a. In the morning of July 26 , wind direction was approximately aligned with array TX. The decay for TX with separation was evidently smaller than that for TY. Wind direction started to shift steadily around noon. At 1500 PST the wind angle was about the same for both arrays, and the decay was almost identical. The model of form (21) describes the observations rather well in general.

In practice, (21) and (32) can be used to assess flux underestimation due to horizontal sensor separation and to correct the measurements. Based on these equations, $H\left(r_{0}\right)$ $\left(r_{0}=3.5 \mathrm{~cm}\right)$ was estimated from the measured $H(\vec{r})$ at various separation distances and the measurements of $\delta$ and $R i_{z}$. The results were then compared with the direct measurements of $H\left(r_{0}\right)$ made with the eddy correlation unit 1138. The relevant information is summarized in Table 2. There exists a good correlation between the estimated and
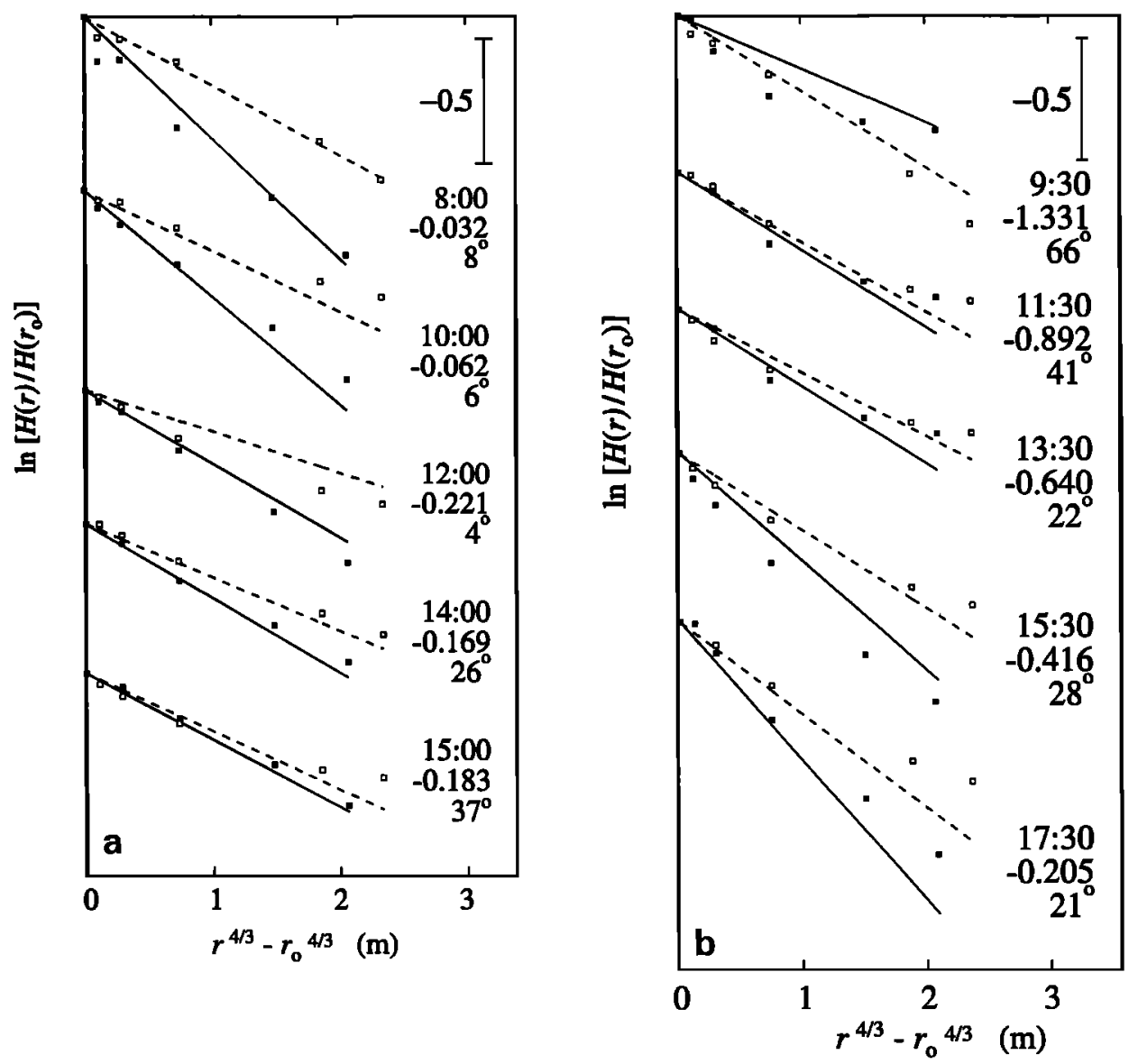

Figure 7. (a) Comparison of the decay of $w-T$ covariance observed (TX, open squares; TY, solid squares) with that calculated using equations (21) and (32) (TX, dashed line; TY, solid line) at $z-d=$ $2.59 \mathrm{~m}$ in the potato field in Delta, July 26, 1992. Also indicated are ending time of the 30-min runs (PST), Richardson number at the height of the covariance measurements, and wind angle for array TX. (b) Same as in Figure 7a except for $z-d=1.54 \mathrm{~m}$ in the clover field, July 31, 1992. 
Table 2. Slope of Regression of $w$-T Covariance $\left(H\left(r_{0}\right)\right)$ Estimated From (21) and (32) for Various Separation Distances ( $r$ ) Against That Measured With Eddy Correlation Unit 1138

\begin{tabular}{|c|c|c|c|c|c|c|c|c|c|c|c|}
\hline & & \multicolumn{10}{|c|}{ Thermocouple Arrays } \\
\hline & & TX & TX & $\mathrm{TX}$ & TX & TX & TY & TY & TY & TY & TY \\
\hline Potato & $r, \mathrm{~m}$ & 0.20 & 0.40 & 0.80 & 1.60 & 1.90 & 0.20 & 0.40 & 0.80 & 1.35 & 1.73 \\
\hline$z-d=2.59 \mathrm{~m}$ & slope & 1.00 & 0.99 & 0.98 & 0.99 & 1.01 & 0.99 & 1.00 & 0.99 & 1.02 & 1.02 \\
\hline$n=\mathbf{3 0}$ & $\boldsymbol{R}$ & 0.993 & 0.986 & 0.981 & 0.969 & 0.961 & 0.986 & 0.985 & 0.981 & 0.967 & 0.952 \\
\hline Clover & $\boldsymbol{r}, \mathrm{m}$ & 0.20 & 0.40 & 0.80 & 1.60 & 1.90 & 0.20 & 0.40 & 0.80 & 1.35 & 1.73 \\
\hline$z-d=1.54 \mathrm{~m}$ & slope & 0.98 & 0.96 & 0.95 & 1.02 & 1.08 & 1.00 & 0.95 & 0.90 & 0.97 & 1.06 \\
\hline$n=32$ & $\boldsymbol{R}$ & 0.995 & 0.994 & 0.990 & 0.965 & 0.943 & 0.994 & 0.994 & 0.984 & 0.973 & 0.938 \\
\hline Clover & $r, \mathrm{~m}$ & 0.20 & 0.40 & 0.80 & 1.60 & 1.90 & 0.20 & 0.40 & 0.80 & 1.35 & 1.65 \\
\hline$z-d=3.01 \mathrm{~m}$ & slope & 0.98 & 0.95 & 0.96 & 0.93 & 0.93 & 0.98 & 0.96 & 0.96 & 0.97 & 0.97 \\
\hline$n=17$ & $R$ & 0.993 & 0.994 & 0.978 & 0.964 & 0.954 & 0.994 & 0.991 & 0.981 & 0.966 & 0.959 \\
\hline
\end{tabular}

Also given are correlation coefficient $(R)$, distance above the displacement height $(z-d)$, and total number of runs $(n)$.

directly measured $H\left(r_{0}\right)$ for all height and separation distance combinations. For 26 of the 30 combinations the agreement was within $\pm 6 \%$.

\section{Discussion}

As shown by the studies of Wyngaard and Cote [1972], Kaimal et al. [1972], and Schmitt et al. [1979] on the vertical velocity and scalar cospectral characteristics in the surface layer under neutral and unstable conditions, the inertial subrange is established beyond $z_{m} k_{1} \cong 3$, where $k_{1}$ is wave number, which is roughly equivalent to $r / z_{m}<0.3$ in the space domain. In this range the $4 / 3$ power law, or the small separation constraint (18) differs from (21) by less than 7 and $3 \%$ at $z_{m} / L=0$ and 1 and $0.5 \%$ at $z_{m} / L=-0.5$ for longitudinal and lateral separations, respectively.

Concern has been raised by a reviewer regarding the assumption $\boldsymbol{H}(\vec{r})=\boldsymbol{H}(-\vec{r})$ (section 3.1). For lateral separation the two covariances should be the same, but for longitudinal separation the mean wind shear may cause these to differ. With the measurement apparatus of the present study (Figure 1), it is not feasible to assess the possible asymmetry of the $w-T$ covariance in the atmospheric surface layer. One alternative is to use $w$ and $T$ time series observed at a single point and apply Taylor's frozen turbulence hypothesis such that $H(r)=P^{-1} \int_{0}^{P} w(t) T(t+r / u) d t$ and $H(-r)=P^{-1} \int_{0}^{P} w(t) T(t-r / u) d t$ for longitudinal separation, where $P$ is the averaging interval and $u$ is the longitudinal velocity component. Taylor's hypothesis is expected to be adequate for $z_{m} k_{1}>3$ (S. Pond, personal communication, 1993). $H(r)$ and $H(-r)$ were then calculated with $r=0.7 \mathrm{~m}$ from the time series obtained by Lee [1992] at $z=2.25 \mathrm{~m}$ over a bare field. For a total of 29 runs there is no appreciable difference between them, as shown by the regression result $H(-r)=0.992 H(r)\left(R^{2}=0.9989\right)$.

Figure 8 compares predictions of (21) and (32) with daytime observations made by Heikinheimo [1986] at two heights over an alfalfa field and averaged over 2 days. The separation was in the lateral direction, with $r_{0}=5 \mathrm{~cm}$ at $z_{m}=8 \mathrm{~m}$ and $r_{0}=20 \mathrm{~cm}$ at $z_{m}=3.5 \mathrm{~m}$. Heikinheimo [1986] did not report stability for his experiment, although from the magnitude of the standard deviation of air temperature $\left(0.3^{\circ}-0.8^{\circ} \mathrm{C}\right)$, it is likely that the atmosphere was moderately unstable. A value of $R i_{z}=-0.2$ was used in our calculation to provide a good fit with Heikinheimo's [1986] observations. This comparison is not a rigorous and inde- pendent validation of (21) and (32), but it confirms the $4 / 3$ scaling law.

Assuming that $3 \%$ is the tolerable flux loss due to sensor separation during eddy correlation measurements, the maximum allowable separation distance $\left(r_{\max }\right)$ will depend on measurement height, stability, and wind direction (Figure 9). There is an advantage in elevating the measurement level: the increase in $z$ will decrease $r_{\max } / z_{m}$ and increase $\left|z_{m} / L\right|$, both resulting in a longer safe separation distance. Of course, when doing so, one should be aware of the fetch requirement.

\section{Conclusion}

The model of $w-T$ spatial covariance, established here for unstable atmosphere in the framework of Monin-Obukhov similarity, describes the observations satisfactorily. The decay of $w-T$ covariance with separation follows $4 / 3$ power law at small separation distances and is exponential at large distances. It is slower in more unstable air and is faster in the lateral direction than in the longitudinal direction. The model can be used to assess the flux underestimation due to sensor separation from information on separation orientation, measurement height, separation distance, and stability. Assum-

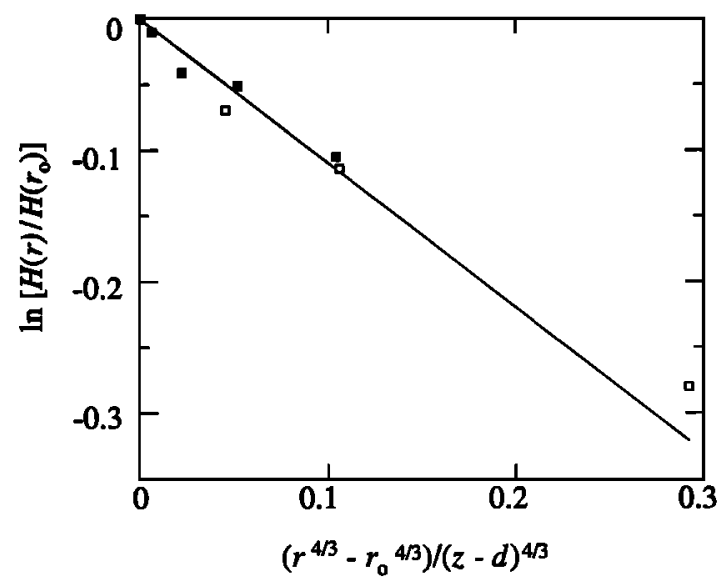

Figure 8. Comparison of the lateral decay of $w-T$ covariance observed by Heikinheimo [1986] (open squares, $z-$ $d=3.5 \mathrm{~m}$; solid squares, $z-d=8 \mathrm{~m}$ ) over an alfalfa field with that calculated from equations (21) and (32) (solid line) using a value of $R i_{z}=-0.2$. 


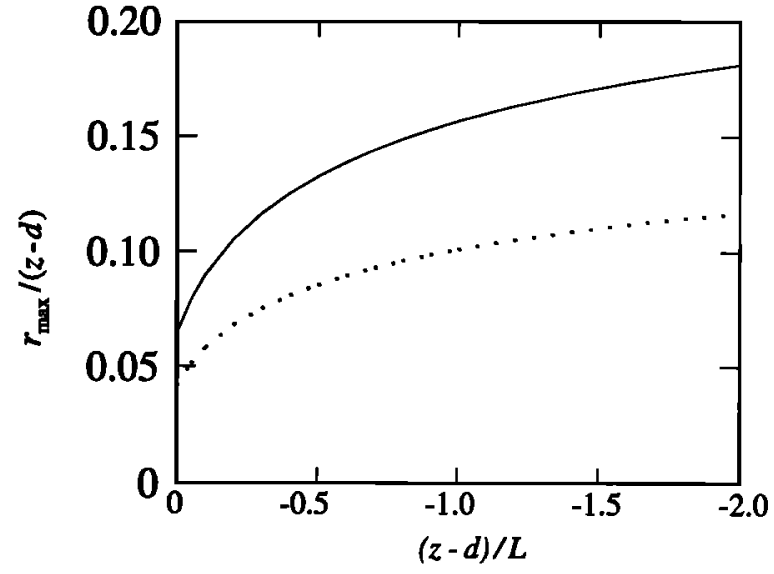

Figure 9. Dependence of the maximum allowable longitudinal (solid curve) and lateral (dotted curve) separation distance $\left(r_{\max }\right)$ on the ratio of height $(z-d)$ to MoninObukhov length scale $(L)$ in the unstable surface layer.

ing that $3 \%$ is an acceptable flux underestimation, a conservative estimate is that the ratio of separation distance to the height above the displacement plane should not exceed about $5 \%$.

In principle, the model can be extended to stable conditions by replacing the similarity functions for sensible heat and turbulent kinetic energy with those for stable air. The experimental verification of this practice is challenging because of the difficulty in measuring fluxes of small magnitude and the very strict requirement of fetch for the site. This issue will be addressed in the future.

Acknowledgments. This research was supported by an operating grant from the Natural Science and Engineering Research Council of Canada. Richard Nistuk provided valuable help in the field. We gratefully acknowledge Hugh and Stan Reynolds for allowing us to use their crop fields. We also thank the two reviewers for their comments on this work.

\section{References}

Arya, S. P., Finite-difference errors in estimation of gradients in the atmospheric surface layer, J. Appl. Meteorol., 30, 251-253, 1991.

Businger, J. A., Transfer of momentum and heat in the planetary boundary layer, in Proceedings of the Arctic Symposium on Heat Budget and Atmospheric Circulation, pp. 305-322, Rand Corporation, Santa Monica, Calif., 1966.

Garratt, J. R., Transfer characteristics for a heterogeneous surface of large aerodynamic roughness, Q.J.R. Meteorol. Soc., 104, 491-502, 1978.

Heikinheimo, M. J., Techniques for determining carbon dioxide and water vapour transport above a vegetated surface using the eddy-correlation method, Ph.D. thesis, Univ. of Guelph, Guelph, Ontario, Canada, 1986.
Hill, R. J., Implications of Monin-Obukhov similarity theory for scalar quantities, J. Atmos. Sci., 46, 2236-2251, 1989.

Hinze, J. O., Turbulence, 2nd ed., McGraw-Hill, New York, 1975.

Jarvis, P. G., G. B. James, and J. J. Landsberg, Coniferous forest, in Vegetation and the Atmosphere, vol. 2, Case Studies, edited by J. L. Monteith, pp. 171-240, Academic, San Diego, Calif., 1976.

Kaimal, J. C., J. C. Wyngaard, Y. Izumi, and O. R. Cote, Spectral characteristics of surface layer turbulence, $Q . J$. R. Meteorol. Soc., 98, 563-589, 1972.

Koprov, B. M., and D. Y. Sokolov, Spatial correlation functions of velocity and temperature components in the surface layer of the atmosphere, Izv. Acad. Sci. USSR Atmos. Oceanic Phys. Engl. Transl., 9, 178-182, 1973.

Kristensen, L., On longitudinal spectral coherence, Boundary Layer Meteorol., 16, 145-153, 1979.

Kristensen, L., and D. R. Fitzjarrald, The effect of line averaging on scalar flux measurements with a sonic anemometer near the surface, J. Atmos. Oceanic Technol., 1, 138-146, 1984.

Kristensen, L., and N. O. Jensen, Lateral coherence in isotropic turbulence in the natural wind, Boundary Layer Meteorol., 17, 353-373, 1979.

Lee, X., Atmospheric turbulence within and above a coniferous forest, Ph.D. thesis, Appendix C, Univ. of B. C., Canada, 1992.

Lee, X., and T. A. Black, Atmospheric turbulence within and above a Douglas-fir stand, II, Eddy fluxes of sensible heat and water vapour, Boundary Layer Meteorol., 64, 364-389, 1993.

Leuning, R., O. T. Denmead, A. R. G. Lang, and E. Ohtaki, Effects of heat and water vapor transport on eddy covariance measurement of $\mathrm{CO}_{2}$ fluxes, Boundary Layer Meteorol., 23, 209-222, 1982.

Moore, C. J., Frequency response corrections for eddy correlation systems, Boundary Layer Meteorol., 37, 17-35, 1986.

Pandolfo, J. P., Wind and temperature for constant flux boundary layers in lapse conditions with a variable eddy conductivity to eddy viscosity ratio, J. Atmos. Sci., 23, 495-502, 1966.

Panofsky, H. A., and J. A. Dutton, Atmospheric Turbulence: Models and Methods for Engineering Applications, John Wiley, New York, 1984.

Schmitt, K. F., C. A. Friehe, and C. H. Gibson, Structure of marine surface layer turbulence, J. Atmos. Sci., 36, 602-618, 1979.

Sellers, W. D., Physical Climatology, University of Chicago Press, Chicago, Ill., 1965.

Silverman, B. A., The effect of spatial averaging on spectrum estimation, J. Appl. Meteorol., 7, 168-172, 1968.

Tatarskii, V. I., The Effects of the Turbulent Atmosphere on Wave Propagation, Kefer, Jerusalem, 1971.

Wyngaard, J. C., and S. F. Clifford, Estimating momentum, heat and moisture fluxes from structure parameters, J. Atmos. Sci., 35, 1204-1211, 1978.

Wyngaard, J. C., and O. R. Cote, Cospectral similarity in the atmospheric surface layer, Q.J.R. Meteorol. Soc., 98, 590-603, 1972.

Wyngaard, J. C., and M. A. LeMone, Behavior of the refractive index structure parameter in the entraining convective boundary layer, J. Atmos. Sci., 37, 1573-1585, 1980.

Wyngaard, J. C., Y. Izumi, and S. A. Collins, Behavior of the refractive-index-structure parameter near the ground, $J$. Opt. Soc. Am., 61, 1646-1650, 1971.

T. A. Black, Department of Soil Science, University of British Columbia, Vancouver, British Columbia, Canada, V6T 1 Z4.

$X$. Lee, School of Forestry and Environmental Studies, Yale University, New Haven, CT 06511.

(Received April 21, 1993; revised December 3, 1993; accepted April 5, 1994.) 